\title{
IBM's PowerEN Developer Cloud: Fertile Ground for Academic Research
}

\author{
Amit Golander, Nancy Greco, Jimi Xenidis, Maria Hyland, Brian Purcell and David Bernstein
}

IBM

\begin{abstract}
IBM's newest technology, the Power Edge of Network (PowerEN) processor, merges network and server attributes to create a new class of wire-speed processor. PowerEN is a hybrid computer that employs: massive multithreading capabilities, integrated $\mathrm{I} / \mathrm{O}$ and unique special-purpose accelerators for compression, cryptography, pattern matching, XML and Network processing.

As a novel architecture, the PowerEN processor offers fertile ground for research. It can facilitate the development of faster applications in many fields of computer science such as Networking, Cryptography, Virtualization, Bioinformatics, SOA, and Systems. IBM encourages academic research and has set up the "PowerEN Developer Cloud" infrastructure to allow it.
\end{abstract}

\section{INTRODUCTION}

Academia is constantly struggling to get access to the latest technology. Researchers look to leverage state-of-theart technology to perform ground-breaking research, often a challenge with yesterdays tools. Students require leading-edge technology in order to build the right skill base for tomorrow's market needs. IBM, as both an industrial and research leader, dedicates attention and resources to address this challenge. One example is the recently announced Academic Cloud [1] that encourages students to develop up-to-date software skills. Another initiative is the PowerEN Developer Cloud, which is the focus of this paper.

Power Edge of Network (PowerEN) is probably the most complex chip IBM has built to date. Targeting a different set of workloads than IBM's traditional System P, PowerEN is targeting the applications at the intersection of where network and servers are now blurring [2]. PowerEN has 58\% more transistor density than traditional P CPUs, using more logic than cache cells (refer to die photo in Figure 1). PowerEN is unique since it is the blurring of Network and Server worlds:

- It has many threads and low-power cores that support a full 64bit PowerPC server ISA, including floating point and virtualization.

- It is designed for ease-of-use, thus standard programming models, operating systems and hypervisors are used.

- It has advanced cache features and small line sizes in order to make more efficient use of scarce memory bandwidth.

- PowerEN's accelerators are designed for both Networking and Application tiers. For instance, they share processor memory space ${ }^{1}$.

\footnotetext{
${ }^{1}$ Network processor accelerators operate on I/O space.
}

- It has integrated on-chip I/O in order to support low power, low latency and high throughput at the same time.

- It supports server-class reliability and RAS.

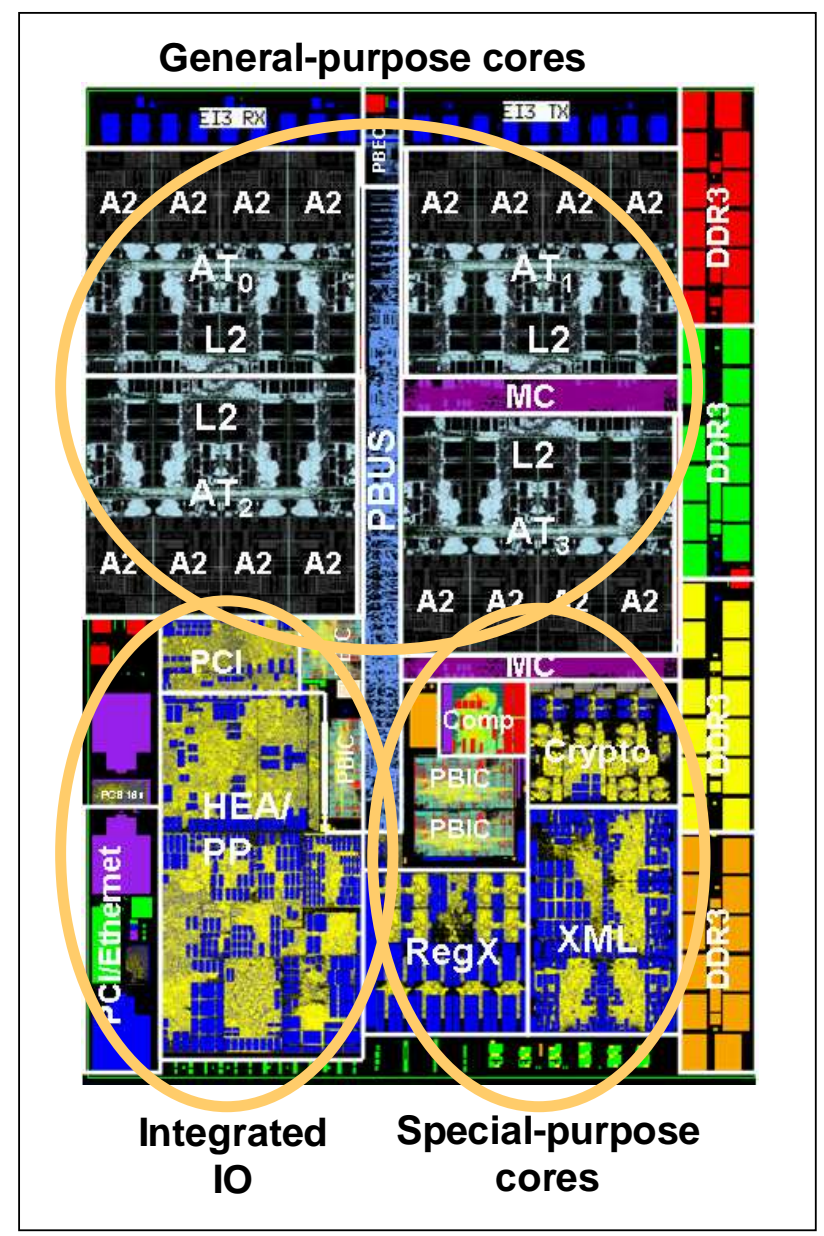

Fig. 1. Full chip die photo of PowerEN

This paper ${ }^{2}$ focuses on the PowerEN Developer Cloud,

${ }^{2}$ Copyright (C) 2010 IEEE. Reprinted from 26-th Convention of Electrical and Electronics Engineers in Israel. This material is posted here with permission of the IEEE. Such permission of the IEEE does not in any way imply IEEE endorsement of any of IBM's products or services. Internal or personal use of this material is permitted. However, permission to reprint/republish this material for advertising or promotional purposes or for creating new collective works for resale or redistribution must be obtained from the IEEE by writing to pubs-permissions@ieee.org. By choosing to view this document, you agree to all provisions of the copyright laws protecting it. 
which is an initiative to provide academia with early access to PowerEN cutting edge technology for research and skill development purposes. The remainder of the paper is organized as follows. Section II describes the PowerEN processor. Section III explains the cloud delivery mechanism chosen for the PowerEN Developer Cloud. Finally, Section IV surveys examples of some research opportunities enabled by the PowerEN technology.

\section{The Power Edge of Network Processor}

PowerEN is designed to enable wire-speed computing in the data center and throughout the network. PowerEN delivers throughput-oriented compute and connectivity density on a single chip. It is mainly constructed of:

- Massive multi-threading (MMT) capabilities - 64 hardware threads per chip and up to 256 coherent threads per system.

- Multiple special-purpose accelerators including XML processing, compression, cryptography, pattern matching (RegX) and TCP/IP/Ethernet acceleration.

- Integrated 10Gbps Ethernet ports as well as PCIe ports, which can be used as both root-complex and end-point modes.

PowerEN has strong on-chip interconnects, two memory controllers, advanced caches and an embedded hypervisor in order to process high-throughput traffic at a very low latency [3].

\section{A. General Purpose Cores}

PowerEN was designed for highly-parallelizable applications, so its architectural focus was on aggregated performance and performance-per-Watt. PowerEN offers up to 64 hardware threads per chip and 256 per a 4-way SMP coherent system. It has a unique hierarchical chip multiprocessor (CMP [4]) structure that efficiently shares cache and interconnect resources [5]. A chip has four compute clusters, each compute cluster is constructed of four 64bit PowerPC general-purpose cores that share an L2 cache. Every core supports simultaneous multithreading (SMT [6]) of four hardware threads, thus $4_{S M T} \times 4_{\text {Cores }} \times 4_{\text {Clusters }} \times 4_{\text {Chips }}=256$ hardware threads.

Designing a server with an unusual number of cores and threads goes beyond replicating core instances. Specific design attention was placed on:

- Ensuring that the off-chip memory access does not become a bottleneck. This was achieved via shared L2 caches, strong chip interconnects, four memory channels and advanced cache features such as cache to cache transfers, cache inject and slave memory.

- Avoiding context switches to hypervisor and operating systems. A PowerEN user space thread has direct access to timers, time-of-day, accelerators, I/O and can directly communicate with other threads without degrading the system security.

- Being able to exploit the vast number of cores to simplify the management of complex tasks such as the deployment of real-time virtual machines and power management.
TABLE I

POWEREN ACCELERATOR Performance

\begin{tabular}{|l|c|c|c|}
\hline Accelerator & $\begin{array}{c}\text { Number } \\
\text { of } \\
\text { engines }\end{array}$ & $\begin{array}{c}\text { Typical } \\
\text { bandwidth } \\
\text { [Gbps] }\end{array}$ & $\begin{array}{c}\text { Peak } \\
\text { bandwidth } \\
\text { [Gbps] }\end{array}$ \\
\hline \hline Pattern matching & 8 & $20-40$ & 72 \\
\hline XML & 4 & $4-12$ & 20 \\
\hline Compression & 1 & 8 & 16 \\
Decompression & 1 & 8 & 16 \\
\hline Cryptography: & 5 & 25 & 40 \\
AES & 8 & 32 & 48 \\
DES & 8 & 16 & 20 \\
TDES & 2 & 2 & 5 \\
ARC4 & 2 & 5 & 6 \\
Kasumi & 6 & $21-24$ & $24-36$ \\
SHA-1,256,512 & 6 & 24 & 30 \\
MD5 & 5 & $10-15$ & $20-30$ \\
AES/SHA & 6 & $9-12$ & 12 \\
TDES/SHA & 3 & $45 \mathrm{~K} \frac{o p}{s e c}$ & $50 \mathrm{~K} \frac{o p}{s e c}$ \\
RSA 1K $\frac{\text { bit }}{\text { key }}$ & 3 & $6 \mathrm{~K} \frac{o p}{s e c}$ & $7.2 \mathrm{~K} \frac{o p}{s e c}$ \\
RSA 2K $\frac{\text { iti }}{\text { key }}$ & 3 & \multicolumn{2}{c}{} \\
\hline
\end{tabular}

\section{B. Special Purpose Cores}

Processors and threads alone are not enough to keep up with wire-speed computing across multiple $10 \mathrm{G}$ ports. PowerEN uses embedded hardware accelerators as a power-efficient way to deliver performance for standard functions:

- Host Ethernet acceleration for network protocol processing

- Encryption / Decryption acceleration

- Pattern Matching acceleration [7]

- XML acceleration [8]

- Compression / decompression acceleration [9]

PowerEN accelerators are designed for high throughput as shown in Table I. They can be activated directly from user space, using virtual memory addresses, which is key for application developers ease of use. Accelerators further support virtual functions to enable quality of service; state preservation ${ }^{3}$ and occasionally complementary software for the more complex XML and RegX accelerators. The accelerators can be used in combination to achieve certain function at very high throughput; for example, achieve $100 \%$ deep packet inspection at $40 \mathrm{Gbps}$, which is a key capability for security workloads.

\section{Integrated $\mathrm{I} / \mathrm{O}$}

PowerEN wire-speed processing is all about quickly moving large amounts of I/O data. Its I/O design is focused on Ethernet as the defacto data-center communication standard and PCIe in order to maintain protocol flexibility and to plug into larger systems. PowerEN has implemented I/O virtualization in all I/O protocols and modes.

Figure 2 demonstrates the very low latency associated with PowerEN integrated Ethernet approach. PowerEN processes Ethernet traffic in a single hop, while traditional compute

\footnotetext{
${ }^{3}$ For example in order to decompress a data stream that arrives in chunks, while allowing the accelerator to process other streams in between.
} 
systems require 3-5 hops. Every hop consumes power and adds delay, especially if protocol translation is required.

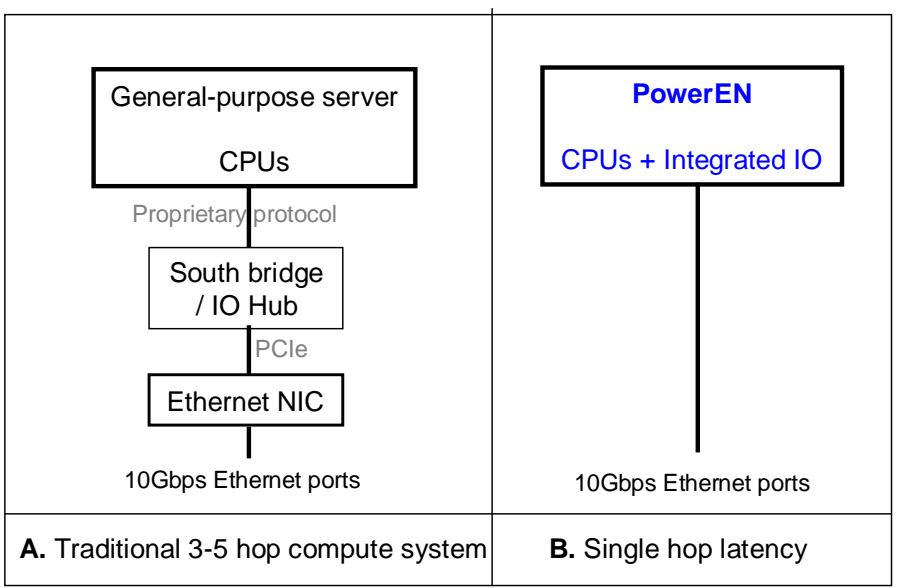

Fig. 2. The latency associated with traditional and PowerEN compute systems

PowerEN Ethernet support includes four integrated 10Gbps as well as two $1 \mathrm{Gbps}$ ports and a packet processing accelerator (HEA). The HEA role is mainly to perform TCP/IP offload and classify the incoming traffic to different queues, so that it can be processed by many hardware threads concurrently [10]. The HEA supports two modes of operation: end-point mode and network-node mode. End-point mode is optimized for transport layer protocol termination, while network-node mode is optimized for port to port packet forwarding.

PowerEN PCIe support includes three Gen2 ports, which can be used as root complex $(8 \mathrm{x}$ and $1 \mathrm{x})$ or end-point $(16 \mathrm{x})$ in the PCIe tree. Figure 3 illustrates PowerEN usage in two compute models: Hybrid computing and Stand alone server. Hybrid computing achieve superior performance and performance-per-watt by matching the best CPU type per task [11]. Stand-alone is the common server type and represents a simpler compute model.

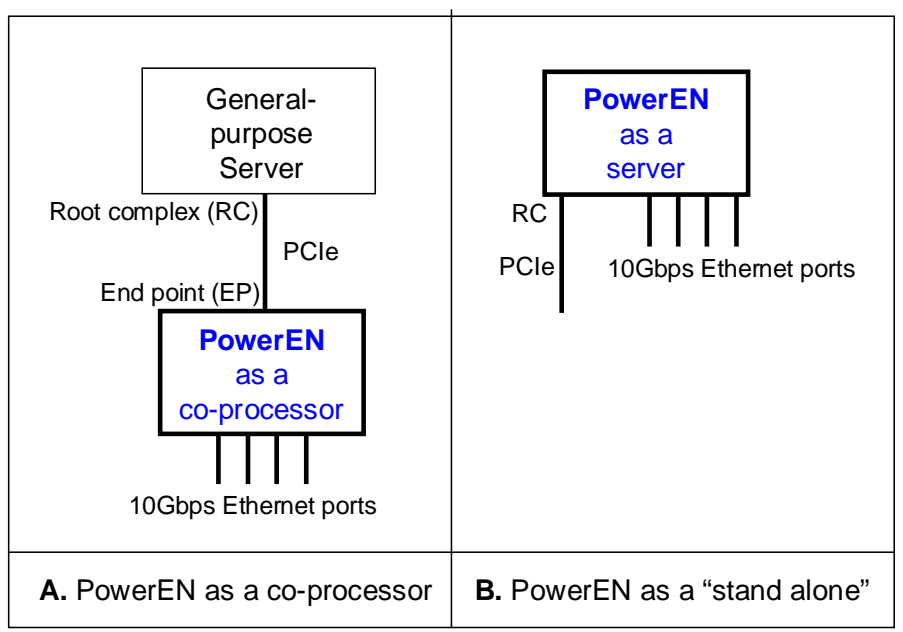

Fig. 3. Hybrid-computing and stand-alone compute models

\section{Poweren Developer Cloud}

IBM, through its university relation programs [12], assists students and academic researchers in their constant struggle to get their hands on the newest technology. Recent years have shown tremendous momentum in cloud infrastructure [13], making it easy to provide the PowerEN platform as a service $(P a a S)$. Our PowerEN PaaS infrastructure, which we call "PowerEN Developer Cloud", is an efficient delivery model for multiple reasons:

1) Fewer boards are required, since hardware is time shared between users.

2) Boards do not have to be shipped out, saving mailing, custom and certification overheads.

3) Software development kit (SDK) is upgraded and maintained by a single administrator.

4) End users benefit from immediate and scalable access.

Obviously, we also plan to distribute evaluation boards in certain cases, for example when unique environments are required.

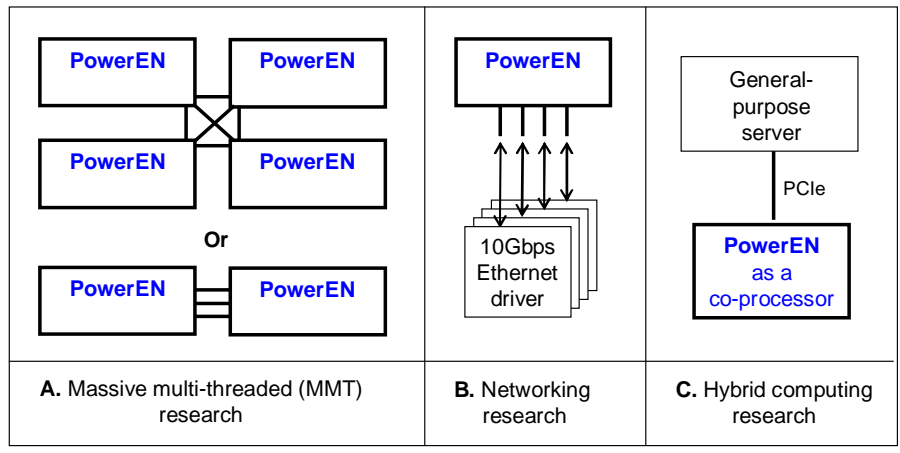

Fig. 4. PowerEN developer cloud configuration examples: A. 256 or 128 hardware thread configuration suited for parallel programming research; B. 10Gbps network drivers configuration suited for networking research; and C. Hybrid computer configuration that is suited for accelerating specific applications.

The PowerEN processor has yet to be released, so our PaaS infrastructure has two major flavors: simulated model [14] and actual hardware. PowerEN simulator is stable, but requires long runtimes. The hardware-based infrastructure is further divided into different configurations. Figure 4 exemplifies three such environments designated for parallel programming, networking and hybrid computing research.

The PowerEN developer cloud infrastructure is at beta phase and available only to selected universities. Currently, to become a user, one must contact an author of this paper ${ }^{4}$. The infrastructure is provided free of charge, but we do ask that you cite this paper in future publications.

\section{EXAMPLES OF RESEARCH OPPORTUNITIES}

A handful of academic research activities have already begun using the PowerEN developer cloud. Proteomics work-

\footnotetext{
${ }^{4}$ amitg@il.ibm.com or maria_hyland@ie.ibm.com
} 
load acceleration [15] using PowerEN's pattern matching accelerator (Tel-Aviv University, Israel), stream-processing systems (Trinity College, Ireland) and High-productivity compute systems (Georgia Tech, USA) are three such world wide examples. Interesting research directions can be found in many domains, as exemplifies in the remainder of this section. Obviously, these directions are just a subset, provided to capture the imagination.

\section{A. Parallel Programming}

The exploitation of process and thread parallelism has been a hot research topic for decades and even more so ever since chip multi-processors became popular [4]. Parallelism needs to be addressed at all levels of the software stack, from the operating system to runtime environments and middleware, to programming languages and applications [16]. Application behavior analysis and development of ground breaking parallelization tools can certainly make use of PowerEN with its 256 hardware threads in a 4-chip SMP configuration.

PowerEN further provides the means for optimizing applications in user space. A multithreaded application can for example schedule its own threads, send inter-thread messages and process Ethernet and PCIe I/O without using system calls and without starving other applications running on other cores. Getting rid of the old habit of optimizing performance by moving software to kernel space does not only better utilize MMT, but also improves system's reliability and eases software development.

\section{B. Networking}

Network bandwidth is increasing at a much higher pace than single thread compute performance. With 40 and 100 Gbps Ethernet ports on the horizon, it is essential to develop multithreaded networking software stacks [17]. PowerEN is a perfect match for such research since it uses standard Linux and built to provide user space threads direct I/O access. Moreover, PowerEN offers advanced ingress processing features, such as queue selection through content or programmable tuples [18] and TCP/IP offload engines [19]. PowerEN Ethernet complex supports two modes: end-point mode which is used for applications that need TCP termination and network mode which is optimized for flow-through traffic.

PowerEN has enough compute power to be used for control plane processing. It can be used to build scalable controllers for architectures such as OpenFlow [20] or DIFANE [21]. PowerEN MMT capability is also a good match for stateless packet processing which is inherently a parallel task. The special-purpose cores can be advantageous for Service plane operations, in which deep packet processing is necessary for applications such as UTM [22], Tunneling, etc. By offloading some of the data/packet operations to the special-purpose cores, the general-purpose cores can be freed up to optimize other time-consuming operations such as packet I/O. Finally, the programmability of PowerEN combined with its wire- speed processing make it a good option to explore Software Routing applications.

\section{Hybrid Computing}

Workload-optimized systems may exploit hybrid computing as a mean to improve their performance and power \& space consumption. PowerEN supports hybrid computing in two dimensions. On chip, it offers a mixture of general- and special-purpose cores. Off chip, PowerEN is unique because it can operate as both a system root complex like general purpose servers and a system end point like a GPGPU [23]. The hybrid-computing model introduces multiple research questions, several examples are:

1) How to best design and program special-purpose accelerators [24].

2) Which frameworks are best suited for hybrid-computing models [25].

3) How to best analyze and present hybrid-computing performance information [26].

Specific accelerator optimization can also be the subject of future research. How to optimize service-oriented architecture (SOA) workloads using a mixture of embedded XML accelerators and threads [8]. How to enhance RegX software heuristics, such as RegX compiler and software cache replacement policy. How should the compression and Cryptographic accelerators be utilized to resolve memory and I/O bound applications or significantly reduce the required storage capacity [27], [28].

Finally, specific hardware functions may shift the implementation tradeoffs of specific workloads. The existence of a RegX accelerator may replace large hash tables, allow semantic canonization of data in flight or significantly speedup applications that are based on pattern matching, such as antiviruses [29]. Using an XML accelerator may make it more cost efficient to store and query XML documents in a database [30] instead of storing preprocessed information in a relational database. As a final example, the ability to define portions of the cache as local memory may be used against side-channel attacks.

\section{Summary AND CONCLUSIONS}

IBM's PowerEN processor delivers throughput oriented compute and connectivity density as well as flexible and scalable resource deployment capability. PowerEN is designed for wire-speed processing in the data center and throughout the network. Nonetheless, its unique characteristics can also be leveraged as fertile ground for academic research. IBM has set up the "PowerEN Developer Cloud" PaaS infrastructure that allows students and faculty members free access to this cutting edge technology.

In this paper we have shared several ideas on how PowerEN can be exploited for academic research in multiple domains, but these are just a few examples. We would like to invite other researchers, domain experts and innovators to come join us in researching, designing and optimizing systems for the next generation of special-purpose systems that industries need. We also welcome your input and recommendations on how to get 
cutting-edge technology into academia faster, easier and more accessible in an open collaborative manner.

\section{ACKNOWLEDGMENTS}

The PowerEN processor, SDK and PaaS infrastructure represent the hard work of many talented IBMers. We would like to thank all of them. We would also like to thank the openminded faculty members we have met so far for their insight.

\section{REFERENCES}

[1] "IBM launches Academic Cloud to speed delivery of technology skills to college students," http://www03.ibm.com/press/us/en/pressrelease/29367.wss, Feb. 2010.

[2] C. Johnson, D. Allen, J. Brown, S. Vanderwiel, R. Hoover, H. Achilles, C. Cher, G. May, H. Franke, J. Xenidis, and C. Basso, "A wire-speed Power processor: $2.3 \mathrm{GHz} 45 \mathrm{~nm}$ SOI with 16 cores and 64 threads," in Proc. of the IEEE Int'l Solid-State Circuits Conference, Feb. 2010.

[3] H. Franke, J. Xenidis, C. Basso, B. Bass, S. Woodward, J. Brown, and C. Johnson, "Introduction to the wire-speed processor and architecture," IBM Journal of Research and Development, vol. 54, no. 1, 2010.

[4] J. Rattner, "Multi-core to the masses," in Proc. of the 14th Int'l Conf. on Parallel Architectures and Compilation Techniques, Sep. 2005, p. 3.

[5] A. Golander, N. Levison, O. Heymann, A. Briskman, M. Wolski, and E. Robinson, "A cost-efficient L1-L2 multicore interconnect: Performance, power and area considerations," IEEE Transactions on Circuits and Systems-I, vol. 58, no. 3, 2011.

[6] S. Eggers, J. Emer, H. Leby, J. Lo, R. Stamm, and D. Tullsen, "Simultaneous multithreading: a platform for next-generation processors," IEEE micro, vol. 17, no. 5, pp. 12-19, 1997.

[7] J. Lunteran, "High-performance pattern-matching for intrusion detection," Apr. 2006, pp. 1-13.

[8] H. Franke, T. Nelms, H. Yu, H. Achilles, and R. Salz, "Exploiting heterogeneous multicore-processor systems for high-performance network processing," IBM Journal of Research and Development, vol. 54, no. 1, 2010.

[9] H. Yu, H. Franke, G. Biran, A. Golander, T. Nelms, and B. Bass, "Stateful hardware decompression in networking environment," Nov. 2008, pp. 141-150.

[10] IBM Redbooks, "Integrated virtual Ethernet adapter technical overview and introduction," Tech. Rep., Oct. 2007.

[11] C. Crawford, P. Henning, M. Kistler, and C. Wright, "Accelerating computing with the Cell broadband engine processor," in Proc. of the 5th Conf. on Computing frontiers, 2008, pp. 3-12.

[12] "IBM university relations," www.ibm.com/developerworks/university/.

[13] G. Gruman, "What cloud computing really means," www.infoworld.com/d/cloud-computing/what-cloud-computing-reallymeans-031, Apr. 2008.

[14] K. Wang, Y. Zhang, H. Wang, and X. Shen, "Parallelization of IBM Mambo system simulator in functional modes," ACM SIGOPS Operating Systems Review, vol. 42, no. 1, pp. 71-76, 2008.

[15] S. Manole, A. Golander, and S. Weiss, "Workload optimization of proteomics pattern matching using embedded accelerator," in Proc. of the 26th IEEE Convention of Electrical \& Electronics Engineers in Israel, Nov. 2010.

[16] D. LaPotin, S. Daijavad, C. Johnson, S. Hunter, K. Ishizaki, H. Franke, H. Achilles, D. Dumarot, N. Greco, and B. Davari, "Workload and network-optimized computing systems," IBM Journal of Research and Development, vol. 54, no. 1, 2010.

[17] http://kernelnewbies.org/Linux_2_6_35.

[18] Z. Zhu, L. Chen, Y. Lin, and L. Shao, "VoIP performance on multicore platforms," IBM Journal of Research and Development, vol. 54, no. 1, 2010.

[19] F. Neeser, B. Metzler, and P. Frey, "SoftRDMA: Implementing iWARP over TCP kernel sockets," IBM Journal of Research and Development, vol. 54, no. $1,2010$.

[20] N. McKeown, T. Anderson, H. Balakrishnan, G. Parulkar, L. Peterson, J. Rexford, S. Shenker, and J. Turner, "OpenFlow: enabling innovation in campus networks," ACM SIGCOMM Computer Communication Review, vol. 38 , no. 2, pp. 69-74, 2010 .
[21] M. Yu, J. Rexford, M. Feedman and J. Wang, "Scalable flow-based networking with DIFANE," in Proc. of the ACM SIGCOMM Conf., Sep. 2010, pp. 351-362.

[22] Y. Qi, B. Yang, B. Xu and J. Li, "Towards system-level optimization for high performance Unified Threat Management," in Proc. of the 3rd Int'l Conf. on Networking and Services, Jun. 2007, p. 7.

[23] D. Luebke, M. Harris, N. Govindaraju, A. Lefohn, M. Houston, J. Owens, M. Segal, M. Papakipos, and I. Buck, "GPGPU: generalpurpose computation on graphics hardware," in Proc. of the 2006 ACM/IEEE Conf. on Supercomputing, Nov. 2006, p. 208.

[24] G. Davis, A. Krishna, S. Ramani, J. Derby, and K. Vu, "A proposal for efficient cpu-accelerator handshake," in Proc. of the 1st ACM ICS Workshop on Accelerators for High-performance Architectures, May 2009.

[25] V. Saraswat, "APGAS: Programming for concurrency and distribution," www.cs.waseda.ac.jp/gcoe/eng/whatsnew/img/080428VSaraswat1APGAS.pdf, Apr. 2008.

[26] M. Biberstein, U. Shvadron, J. Turek, B. Mendelson, and M. Chang, "Trace-based performance analysis on Cell BE," in IEEE Int'l Symp. on Performance Analysis of Systems and Software, Apr. 2008, pp. 213-222.

[27] "Opendedup an open source deduplication file system," www.opendedup.org.

[28] "Primary storage optimization: an overview," www.storwize.com/Library_WP.asp.

[29] "ClamAV an open source GPL anti-virus toolkit," www.clamav.net/lang/en/.

[30] M. Nicola, and P. Kumar-Chatterjee, "DB2 pureXML cookbook: master the power of the IBM hybrid data server," in IBM Press, 2009. 